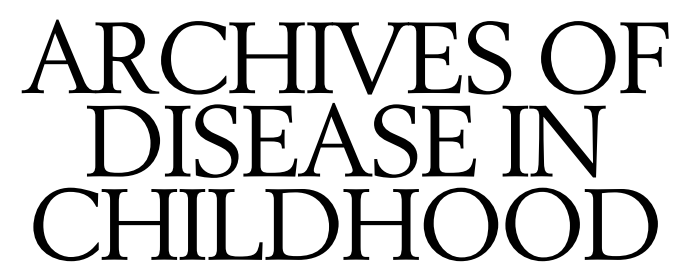

The fournal of the Royal College of Paediatrics and Child Health

\title{
Telemedicine in paediatric cardiology
}

Telemedicine can broadly be defined as the application of modern telecommunication systems for clinical purposes. The essential feature is that there is remote consultation: the doctor and patient are in different places often separated by large distances. Such remote consultation usually requires the transfer of images from the referral centre to a centre of expertise.

\section{The technology}

The explosion in the number of telemedicine projects is largely due to major advances in telecommunications technology, which have made it possible to transfer large amounts of data quickly. One of the most significant developments is the introduction of integrated services digital network (ISDN) lines to the telephone network of most western countries. These lines have the capacity to allow rapid transfer of the large amount of information necessary to transmit moving images such as ultrasound pictures. Before transmission the image is converted to a digital signal by a "codec", which is usually a desktop videoconferencing unit. Transmission is then possible using ISDN, fibreoptic, radiowave or even satellite networks. After transmission a second codec at the receiving end reverses the process reconstructing the image. The information carrying capacity of any telecommunication system is measured in terms of bandwidth, the unit of which is bits/ second. The technological sophistication of systems ranges from the standard analogue telephone line (38 kbits/ second) to national broadband networks such as the British universities superJANET system with a bandwidth of $600 \mathrm{Mbits} / \mathrm{second}$. Two ISDN lines have a bandwidth of $128 \mathrm{Kbits} / \mathrm{second}$.

\section{Application to other specialties}

Telemedicine lends itself particularly to those specialties where images are crucial to diagnosis. Thus there have been many applications in radiology with remote reporting of radiographs, computed tomography, and magnetic resonance images. ${ }^{1-3}$ In pathology remote reporting on frozen sections and other histological specimens has been successfully performed. ${ }^{4}$ Recently, remote consultation on fetal ultrasound images has been reported. ${ }^{5}$ In cardiology the simplest applications are in the transmission of electrocardiograms (ECG). ${ }^{6}$

\section{Application to paediatric cardiology}

The major application in paediatric cardiology is in the transmission of echocardiographic images to provide remote diagnosis. This is particularly relevant to the diagnosis of congenital heart disease (CHD) in the neonate, where early diagnosis and institution of appropriate management may be crucial to the outcome for the patient. Many such babies are born in district general hospitals where there is not immediate access to a paediatric cardiologist, thus transfer to a paediatric cardiology centre is usually necessary. The main benefit of a telemedicine link is to be able to provide a remote diagnosis from transmitted echocardiographic images before deciding whether patient transfer is indicated. There is a double benefit in that those with serious CHD are diagnosed and transferred early and those in whom CHD is excluded are not subjected to an unnecessary and potentially hazardous journey. This is obviously particularly important where the units are separated by long distances as has been shown by Finlay et al in their work in Nova Scotia, Canada. ${ }^{78} \mathrm{We}$ have also shown in Northern Ireland that less expensive systems can be used to provide accurate remote diagnosis. ${ }^{9}$ Figure 1 summarises the components of the system we now regularly use in clinical service.

Some of the major considerations in establishing a telemedicine link for transmission of echocardiograms are:

- Who performs and transmits the echocardiograms: technician or doctor?

- What training in paediatric echocardiography is necessary before participation in a link?

- Who receives the images and provides the expert opinion?

- Who to link to?

- What is the available budget and who pays?

Altnagelvin neonatal unit

RBHSC paediatric cardiology

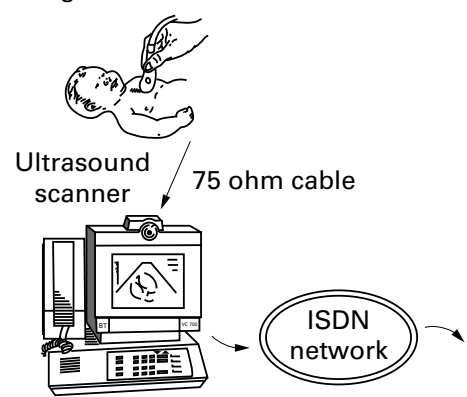

BT VC 7000 unit

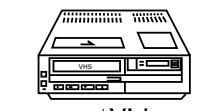

AVideo recorde

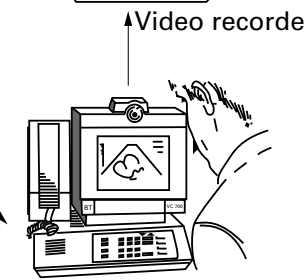

BT VC 7000 unit
Figure 1 Telemedicine system for transmission of echocardiograms. Altnagelvin is a district general hospital in Northern Ireland. RBHSC, Royal Belfast Hospital for Sick Children. 


\section{The echocardiographer}

In most reported studies of transmission of paediatric echocardiograms, technicians have performed the scans, whereas our experience has been with consultant paediatricians in the neonatal unit. ${ }^{10}$ The skills of the medical staff in ultrasound and the availability of technical staff, particularly out of normal working hours, will obviously influence the choice in a particular district general hospital. Before participating in a telemedicine link it is highly desirable that staff who are to perform the scans should have at least attended a basic course in paediatric echocardiography. In a recent article Skinner addressed the issue of echocardiography on the neonatal unit. ${ }^{11} \mathrm{He}$ suggested that one neonatologist in each unit should have some experience in echocardiography. I broadly agree, but there are obvious potential dangers in personnel with limited experience in echocardiography and paediatric cardiology performing studies to exclude CHD. A telemedicine link can provide the ideal situation with the scan being performed by a neonatologist with the help of "live" guidance by a paediatric cardiologist.

\section{The "expert"}

A consultant paediatric cardiologist usually interprets transmitted images. Live transmission is preferable to transmission of prerecorded studies as it allows the paediatric cardiologist to guide the person performing the scan so that all the views necessary to reach a diagnosis are obtained. We have also found that one of the benefits of the telemedicine link is an educational one with the echocardiography skills of the paediatricians improving over our two year study period.

\section{Who to link to?}

Most links are likely to be to district general hospitals with paediatric and neonatal units. The provision of this service, if links are established to a number of peripheral hospitals, is time consuming as each study can take up to an hour. Preference should therefore be given to centres most remote from access to a paediatric cardiology unit.

\section{Financial considerations.}

Computer based desktop videoconferencing units cost between $£ 5000$ and $£ 10000$ (US\$8-16 000). Further costs in operating a telemedicine link depend on the mode of transmission. Installation costs of each set of two ISDN lines from British Telecom are currently approximately $£ 200$ (US\$320) and rental tariffs are in the region of $£ 150$ to $£ 200$ per quarter (US\$240-320). Remote diagnosis of major lesions is possible with ISDN2 but ISDN6 may be the best compromise between achieving good image quality and limiting costs. The call charges for ISDN lines are similar to those for standard telephone lines. A system using ISDN6 is still relatively inexpensive and purchasers can easily recover costs, by saving on the number of ambulance transfers of neonates to regional centres for a paediatric cardiology opinion. In areas where there are fibreoptic links, such as from cable telephone companies, these can provide an extremely high quality link if a suitable contract can be agreed. Our experience has been that most transmitted studies take 30 to 45 minutes. For a regional centre linked to several peripheral units, considerable time can be spent interpreting and guiding transmitted echocardiograms. Staffing costs, therefore, need to be considered in calculating the annual cost of operating the service.

\section{Risk $v$ benefit}

The earlier diagnosis and initiation of appropriate treatment that can be achieved using telemedicine links should reduce morbidity and mortality but as yet there are no published controlled trials measuring this benefit. A further significant benefit is that unnecessary transfers are avoided. In our series of 63 cases the need for transfer to obtain a diagnosis was avoided in $47 .{ }^{10}$ There are therefore potentially significant financial savings for the referring hospital.

For the paediatric cardiologist the concern about participating in diagnosis by telemedicine is that a serious lesion may be missed and false reassurance given. The medicolegal aspects of patient responsibility after remote consultation in this way are still unclear. In our series of 61 transmitted studies using ISDN2 there were four errors in diagnosis ${ }^{9}:$ three small ventricular septal defects and one moderate pulmonary valvar stenosis were not detected. Twenty eight major lesions were accurately diagnosed. In the series reported by Finlay et $a l,{ }^{8} 26$ patients in whom a diagnosis was made by using their telemedicine link had follow up echocardiography by "paediatric cardiology echocardiography staff”. No significant diagnostic discrepancies were found. Sobczyk et al reported their experience of remote diagnosis in 47 patients from prerecorded images transmitted transtelephonically ${ }^{12} ; 24$ studies were normal. Lesions accurately diagnosed included persistent ductus arteriosus (PDA) $(n=8)$, ventricular septal defect $(n=6)$, and ventricular septal defect with interrupted aortic arch $(\mathrm{n}=1)$. They reported an inappropriate clinical decision causing delay in transfer of a patient with a PDA requiring ligation due to an inconclusive transmitted study.

The available evidence thus suggests that remote echocardiography can be used to diagnose or exclude major lesions with accuracy. The diagnosis made on the transmitted scan should not, however, be considered the definitive cardiac consultation. This is not an issue for patients transferred to the regional centre on the basis of the remote consultation as they will all have a "hands on" consultation and echocardiography. I would advocate that babies considered to be normal or to have minor lesions on the transmitted scan should also electively have a follow up direct consultation by a paediatric cardiologist.

Common diagnostic difficulties for the neonatologist In terms of life or death of the patient, the defects that must be diagnosed are those causing cyanotic heart disease, major obstructive lesions of the left or right heart, and total anomalous pulmonary venous drainage (TAPVD). Cyanotic lesions such as transposition of the great arteries, pulmonary or tricuspid atresia, and other complex lesions with univentricular hearts do not usually present diagnostic difficulty if standard views can be obtained during the transmitted echocardiography. The diagnosis of TAPVD is the one likely to provide the most difficulty and is obviously a crucial one to make, particularly in babies where the differential diagnosis is persistent pulmonary hypertension. In our series, to date there has been only one patient with TAPVD. The correct diagnosis of supracardiac TAPVD was made with the transmitted scan, on which the pulmonary venous confluence and the ascending vein could be seen. The exclusion of a diagnosis of TAPVD requires a combination of imaging the pulmonary veins entering the left atrium and the demonstration of normal Doppler flow from them into left atrium. In the cases to date where a diagnosis of persistent pulmonary hypertension was being considered we have been able to satisfy ourselves that pulmonary venous drainage was normal.

For those with limited experience in echocardiography, obtaining good aortic arch views is one of the areas that causes most difficulty. The diagnosis of coarctation of aorta is essentially a clinical one, and if the femoral pulses are weak or absent in association with an arm-leg blood 
pressure gradient, the patient will require transfer to the regional centre for a definitive diagnosis.

The other common diagnostic dilemma for the neonatologist is in deciding whether the premature infant has a PDA, and, if there is one, how much is it contributing to the clinical condition of the patient? It is not practical to use a telemedicine link to confirm or exclude a PDA in all premature infants with this clinical problem. There is a case, however, for transmitting scans on ventilator dependent babies with chronic lung disease to determine whether a significant shunt through a PDA is exacerbating the problem. It is very useful in this situation to be able to answer the question by remote echocardiography and avoid transferring the ventilated patient.

\section{Conclusions}

It is likely that with further improvements in, and increasing availability of the equipment necessary to transmit good quality echocardiographic images, an increasing number of telemedicine links will be established from neonatal units to paediatric cardiology centres. I believe that it is not appropriate or necessary to apply this technology to the assessment of the child where an urgent clinical decision does not need to be made such as the evaluation of a murmur. Telemedicine links should be used to diagnose or exclude significant neonatal CHD and I think that over the next few years paediatric cardiologists will find that providing remote diagnosis in this way could become an increasingly important part of their workload.
I thank my colleagues Brian Craig and Connor Mulholland for their review of this article and helpful comments.

F A CASEY

Consultant Paediatric Cardiologist,

Royal Belfast Hospital For Sick Children,

180 Falls Road, Belfast BT12 6BE, UK

1 Wright R, Loughrey C. Teleradiology. BMf 1995;310:1392.

2 Barnitzky S, Rosenthal SJ, Siegel EL, et al. Teleradiology: assessment. Radiology 1990;177:11-17.

3 Binkhuysen F, Ottes FP, Valk J, et al. Remote expert consultation for MRI procedures by means of teleradiology. Eur $\mathcal{F}$ Radiology 1995;19:147-50.

4 Nordum I, Engum B, Rinde E, et al. Remote frozen section service: a telepathology project in northern Norway. Hum Pathol 1991;22:514-18.

5 Fisk NM, Bower S, Sesulveda W, et al. Fetal telemedicine: interactive transfer of real-time ultrasound and video via ISDN for remote consultation. 7ournal of Telemedicine and Telecare 1995;1:38-44.

6 Ong K, Chia P, Ng WL, et al. A telemedicine system for high quality transmission of paper electrocardiogram reports. Fournal of Telemedicine and Telecare $1995 ; 1 ; 27-33$.

7 Finlay JP, Human DG, Nanton MA, et al. Echocardiography by telephone: evaluation of pediatric heart disease at a distance. Am $\mathcal{f}$ Cardiol 1989;63:1475-7.

8 Finlay JP, Sharrat GP, Nanton MA, et al. Echocardiography by telemedicine: nine years experience. Fournal of Telemedicine and Telecare 1997;3:200-4.

9 Casey F, Brown D, Corrigan N, et al. Value of a low-cost telemedicine link in the remote echocardiographic diagnosis of congenital heart defects. fournal of Telemedicine and Telecare 1998;4(suppl 1):46-8.

10 Mulholland HC, Casey F, Brown D. Application of a low cost telemedicine link to the diagnosis of neonatal congenital heart defects by remote consultation. Heart. [In press.]

11 Skinner JR. Echocardiography on the neonatal unit; a job for the neonatologist or the cardiologist? Arch Dis Child 1998;78;401-2.

12 Sobczyk WL,Solinger RE, Rees AH. Transtelephonic echocardiography: successful use in a tertiary pediatric referral centre. F Paediatrics 1993;122: S84-8. 